\title{
Produção de mudas e avaliação do crescimento inicial em campo de Amburana cearensis (Allemão) A.C. Smith
}

\section{Seedling production and evaluation of the initial growth in fields of Amburana cearensis (Allemão) A.C. Smith}

DOI: $10.46814 / 1 a j d v 2 n 5-009$

Recebimento dos originais: 10/07/2020

Aceitação para publicação: 30/08/2020

\author{
Danilo Diego de Souza \\ Mestre em Botânica pela Universidade Federal Rural de Pernambuco - UFRPE \\ Instituição: Agência Municipal de Meio Ambiente de Ouricuri-PE - AMMA \\ Endereço: Praça Padre Francisco Pedro da Silva, 145, CEP 56200-000, Ouricuri-PE, Brasil \\ E-mail: danilodiegos@hotmail.com \\ Ana Valéria Vieira de Souza \\ Doutora em Agronomia pela Universidade Estadual Paulista Júlio de Mesquita Filho - UNESP \\ Instituição: Embrapa Semiárido \\ Endereço: Rodovia BR-428, Km 152, s/n - Zona Rural, CEP 56302-970, Petrolina-PE, Brasil \\ E-mail: ana.souza@embrapa.br
}

\begin{abstract}
RESUMO
Amburana cearensis é uma árvore típica da Caatinga e apresenta importância comercial, devido às suas várias aplicações, principalmente para uso madeireiro e medicinal. $\mathrm{O}$ aumento na demanda pelo uso de seus recursos representa uma séria ameaça à sobrevivência, pois esta espécie sofre risco de extinção. Objetivou-se com este trabalho produzir mudas e avaliar o crescimento inicial de mudas de A. cearensis, a fim de caracterizar ecologicamente a espécie e também fornecer subsídios para o desenvolvimento de estratégias para sua conservação. Se faz necessário o conhecimento básico da germinação de sementes, produção de mudas e processos de dinâmica de crescimento de espécies nativas da Caatinga, para que se tenha um manejo correto das florestas nativas. As sementes de $A$. cearensis apresentaram um alto índice de germinação, com $87,69 \%$ e boa formação de mudas. No entanto, a espécie apresentou um crescimento lento, com 34,83 cm de média de altura e 4,92 mm de média de diâmetro do colo aos 12 meses de idade. $\mathrm{O}$ alto índice de germinação, com facilidade na formação de mudas, ajudará na conservação deste recurso genético com atividades florestais para o manejo sustentável e programas para conservação da espécie.
\end{abstract}

Palavras-chave: Caatinga, Germinação de sementes, Conservação.

\begin{abstract}
Amburana cearensis is a typical Caatinga tree and is of commercial importance due to its various applications, mainly for timber and medicinal use. Increasing demand for resource use poses a serious threat to survival, as this species is at risk of extinction. The objective of this work was to produce seedlings and evaluate the initial growth of A. cearensis seedlings, in order to ecologically characterize the species and also to provide subsidies for the development of conservation strategies. It is necessary the basic knowledge of seed germination, seedling production and growth dynamics processes of native Caatinga species, in order to have a correct management of the native forests. A. cearensis seeds presented a high germination index, with $87.69 \%$ and good seedling formation.
\end{abstract}


However, the species presented a slow growth, with a $34.83 \mathrm{~cm}$ mean height and $4.92 \mathrm{~mm}$ mean neck diameter at 12 months of age. The high germination index, with ease in the formation of seedlings will help in the preservation of this genetic resource with forest activities for the sustainable management and programs for conservation of the species.

Keywords: Caatinga, Seed germination, Conservation.

\section{INTRODUÇÃO}

A espécie Amburana cearensis pertencente à família Fabaceae, é uma árvore típica da Caatinga, conhecida popularmente como umburana-de-cheiro, imburana-de-cheiro, cumaru, entre outras denominações. Apresenta importância comercial dada às suas várias aplicações, para uso madeireiro, medicinal, ornamental e forrageiro (LORENZI; MATOS, 2008; MAIA, 2004). Esta espécie ocorre nas regiões secas do Brasil, especialmente na Caatinga. A distribuição desta espécie no Brasil vai desde a Caatinga até a floresta pluvial do vale do Rio Doce, incluindo também os afloramentos calcários e matas decíduas do Centro-Oeste e Sudeste do país (MAIA, 2004).

A madeira, devido à reconhecida durabilidade natural, é utilizada na fabricação de móveis, caixotarias, portas e janelas. As sementes são utilizadas como aromatizante e repelente de insetos (LORENZI; MATOS, 2008; MAIA, 2004; LORENZI, 2008). Na medicina popular, as cascas e sementes são utilizadas no tratamento de doenças respiratórias, como asmas, bronquites, gripes e resfriados (CANUTO et al., 2012). Apresenta também atividade antinociceptiva e anti-inflamatória (OLIVEIRA et al., 2009; LEAL et al., 2011; LIMA et al., 2013) e ação antibacteriana (FIGUEREDO et al., 2013).

A crescente demanda pelo uso dos recursos madeireiros e medicinais de A. cearensis por meio de atividade extrativista predatória, representa uma séria ameaça à sobrevivência da espécie, visto que segundo Brasil (2008), esta espécie sofre risco de extinção.

De acordo Maia (2004), esta espécie pode ser utilizada como componente em reflorestamentos mistos, ambientais e/ou comerciais, em enriquecimento de capoeiras e áreas de Caatinga degradada. Além disso, pode também ser empregada para reposição de mata ciliar, na composição de quebra-ventos, entre outros.

A rapidez e uniformidade na germinação de sementes e formação de plântulas são características desejáveis na produção de mudas, pois quanto mais longo for o tempo que a plântula permanece na fase inicial de desenvolvimento, mais tempo fica sujeita aos fatores adversos do ambiente (MARTINS et al., 1999). No entanto, a germinação lenta, por sua vez, pode gerar o 
aumento nos custos de produção, pois há um maior tempo de permanência nos viveiros para a obtenção de mudas (GUEDES et al., 2010a).

Considerando que estudos básicos de germinação e produção de mudas de espécies nativas da Caatinga são de extrema importância para o desenvolvimento de atividades florestais e para programas de conservação (DANTAS et al., 2011), o conhecimento sobre tais fatores (germinação de sementes, produção de mudas e taxa de crescimento) são medidas importantes para contribuir com um manejo florestal adequado e garantir a conservação de espécies vegetais em risco de extinção.

A avaliação do crescimento da vegetação para caracterização ecológica é de fundamental importância para estudos de sucessão ecológica. Portanto, o conhecimento das características de crescimento e desenvolvimento de espécies vegetais é um fator decisivo no manejo florestal para fins de conservação e uso sustentável, pois permite o uso técnicas mais adequadas para cada tipo de espécie.

Neste contexto, objetivou-se com este trabalho avaliar a produção de mudas e o crescimento de mudas da espécie A. cearensis após um ano de plantio em Banco Ativo de Germoplasma, a fim de caracterizar ecologicamente a espécie, assim fornecer informações básicas para o desenvolvimento de estratégias para sua conservação, consequentemente amenizar o risco de erosão genética.

\section{MATERIAL E MÉTODOS}

Para produção de mudas, as sementes de A. cearensis foram coletadas em 65 árvores de ocorrência espontânea situadas nos municípios de Petrolina-PE, Lagoa Grande-PE e Juazeiro-BA (Figura 1a). As plantas matrizes foram georeferenciadas com uso de aparelho GPS (Global Positioning System). Após a etapa de coleta, as sementes foram levadas ao laboratório de Biotecnologia vegetal da Embrapa Semiárido, Petrolina-PE, onde foi realizado o seu beneficiamento, eliminando-se a ala membranosa (Figura 1b), pois as sementes são dotadas dessa adaptação que facilita a sua dispersão pelo vento. Vale salientar que as sementes não foram armazenadas após o beneficiamento.

O trabalho foi dividido em duas etapas, a primeira foi realizada no viveiro florestal da Embrapa Semiárido, sob sombreamento de 50\% (Sombrite). No viveiro, realizou-se a semeadura em duplicata de cada acesso (semente de cada planta matriz) com o hilo posicionado lateralmente e na profundidade de aproximadamente $1 \mathrm{~cm}$, em sacos de polietileno $\left(10 \times 20 \mathrm{~cm}-650 \mathrm{~cm}^{3}\right)$, contendo mistura de solo + substrato comercial (2:1), respectivamente, totalizando 130 sementes para avaliação da germinação. As sementes não passaram por nenhum tratamento físico ou químico. A 


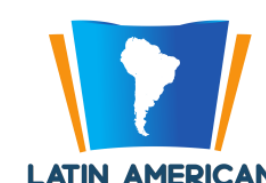

LATIN AMERICAN
JOURNAL OF DEVELOPMENT

emergência foi avaliada diariamente. Calculou-se o percentual de germinação e a frequência relativa de germinação.

A produção de mudas foi conduzida no viveiro florestal, no qual foram irrigadas em sistema de irrigação por microaspersão diariamente. As mudas foram mantidas no interior do viveiro durante um período de noventa dias. Após esse período, estas foram transferidas para o campo, numa área experimental da Embrapa Semiárido, com finalidade de implantação do Banco Ativo de Germoplasma (BAG), sendo plantados dois indivíduos de cada acesso (130 mudas) em espaçamento $5 \mathrm{~m}$ x $5 \mathrm{~m}$. O plantio ocorreu durante o período chuvoso para auxiliar no índice de sobrevivência das mudas.

A segunda etapa foi realizada no Banco Ativo de Germoplasma, no qual avaliou-se, após um ano de implantação do BAG, o crescimento das mudas, sendo feita uma avaliação do crescimento, medindo-se a altura $(\mathrm{cm})$ (comprimento do caule) com uma trena (Figura 1c) e o diâmetro do colo (mm) com um paquímetro digital (Figura 1d). Também foi feito um levantamento da taxa de mortalidade dos acessos durante esse período de um ano. Calculou-se a média aritmética, desvio padrão e coeficiente de variação $(\mathrm{CV})$ para o parâmetro taxa de crescimento das mudas.

Figura 1 - (a) Coleta de sementes de A. cearensis. (b) Beneficiamento de sementes de A. cearensis. (c) Avaliação do comprimento do caule de mudas de A. cearensis. (d) Avaliação do diâmetro do caule de mudas de A. cearensis.

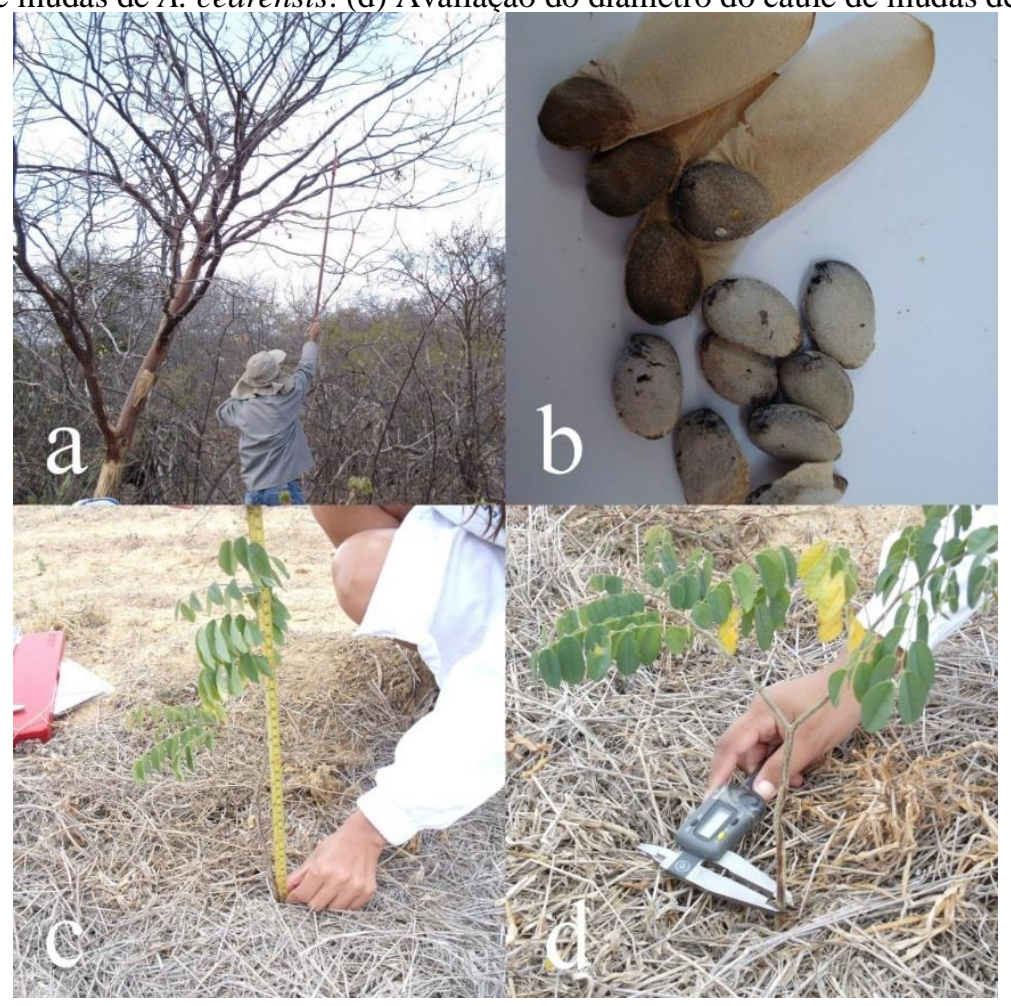

Fonte: Autores. 


\section{RESULTADOS E DISCUSSÃO}

Os resultados mostraram que as sementes de Amburana cearensis alcançou um índice germinação de 87,69\% (114 sementes) após 30 dias da semeadura e que a germinação teve início 7 dias após o início do experimento (Figura 2).

Figura 2 - Frequência relativa da germinação de sementes de Amburana cearensis.

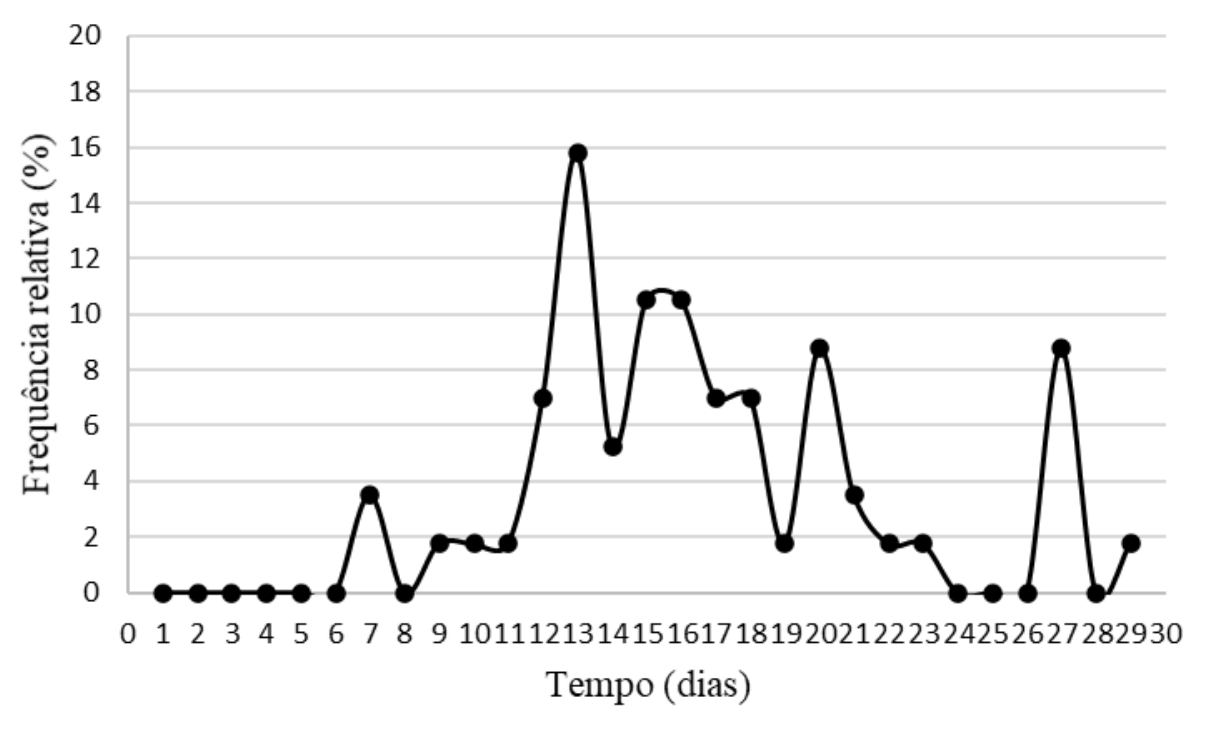

Observa-se que as sementes de Amburana cearensis apresentam um bom índice de germinação, não necessitando de tratamentos físico-químicos, fato esse que evidencia que não há necessidade de se romper a estrutura do tegumento das sementes para indução da germinação, o que evidencia que a semente não apresenta dormência.

O percentual de germinação de Amburana cearensis é relatado por vários autores, demonstrando que a germinação de sementes da espécie apresenta bons resultados, pois as sementes desta espécie não apresentam dormência tegumentar.

Em relação a germinação de sementes, tal estudo corrobora com Lorenzi (2008) e Maia (2004), que citam que a germinação das sementes dessa espécie, inicia-se em 5 a 30 dias e tem um alto poder germinativo, sendo geralmente superior a $80 \%$ para sementes recém-colhidas.

Guedes et al. (2010b) avaliaram a emergência de plântulas de Amburana cearensis acondicionadas em embalagem de papel alumínio e armazenadas em laboratório, e após 270 dias de armazenamento apresentou de $68 \%$ de índice de germinação. Em pesquisa realizada por Lopes et al. (2014), estes constataram 94\% de germinação de sementes de A. cearensis. Angelim et al. (2007) concluíram que a germinação de sementes da espécie foi superior a 70\% quanto imersas em água. Já 
em estudo realizado por Almeida et al. (2014), estes observaram uma porcentagem de germinação de $67 \%$.

Guedes et al. (2013) e Guedes et al. (2015) constataram porcentagens de germinação de 66\% a 98\% coletadas de árvores matrizes em várias localidades distintas na Paraíba, Pernambuco e Rio Grande do Norte. Oliveira et al. (2014) verificaram que as sementes dessa espécie apresentam maior porcentagem de germinação a $30^{\circ} \mathrm{C}$. Após 27 dias de semeadura, constatou-se $96 \%$ de sementes germinadas para a temperatura de $30^{\circ} \mathrm{C}$. Dessa forma, os maiores valores de germinação das sementes em temperaturas elevadas podem estar associados à adaptabilidade da espécie aos fatores ambientais da Caatinga.

Em relação ao crescimento das mudas de A. cearensis, após um ano de implantação do BAG, foi verificado um crescimento lento da espécie, sendo $34,83 \mathrm{~cm}$ de média de altura e 4,92 $\mathrm{mm}$ de média de diâmetro do colo (Tabela 1).

Tabela 1 - Taxa de crescimento de mudas Amburana cearensis após um ano.

\begin{tabular}{c|c|c|c|c|c}
\hline Parâmetro & Valor mínimo & Média & Valor máximo & $\begin{array}{c}\text { Desvio } \\
\text { padrão }\end{array}$ & CV (\%) \\
\hline Comprimento em altura $(\mathrm{cm})$ & 17,00 & 34,83 & 57,5 & 11,29 & 32,42 \\
\hline Diâmetro do colo $(\mathrm{mm})$ & 2,66 & 4,92 & 9,50 & 1,61 & 32,75 \\
\hline
\end{tabular}

Apesar da semente de A. cearensis ter um bom potencial germinativo, vale salientar que seu desenvolvimento no campo é lento. Segundo Carvalho (2003), a frutificação da espécie em plantios ocorre em 10 anos. Dessa forma, foi observado que o crescimento das mudas de A. cearensis, após um ano de implantação do BAG, é lento, corroborando com Lorenzi (2008), quando o mesmo constata que o crescimento da espécie não chega a 1,5 metros aos 2 anos.

De fato, Ramos et al. (2004) estudando a espécie Amburana cearensis em condições de viveiro na Estação Experimental da Universidade de Brasília, verificaram que aos 12 meses de idade a espécie apresentou média de altura a pleno sol de 17,02 cm e aos 14 meses apresentou diâmetro do coleto a pleno sol de $4,12 \mathrm{~mm}$.

Ainda, em relação ao crescimento da espécie, de acordo com Carvalho (2003), em experimentos realizados no estado do Paraná, na cidade de Foz do Iguaçu-PR, aos 9 anos de idade, as plantas cresceram em média 3,0 metros e média do DAP (Diâmetro à altura do peito, medido a 1,30 m do solo) de 4,0 cm. No mesmo município, sendo que em outra área, o crescimento da espécie aos 9 anos de idade chegou a 4,61 metros e DAP de 7,5 cm. Ainda segundo o autor, na cidade de Santa HelenaPR, foi constatado aos 6 anos de idade, uma média de crescimento de 3,66 metros e média do DAP 
de $5,3 \mathrm{~cm}$. E no mesmo município, porém em outra área experimental, o crescimento aos 9 anos após o plantio foi de 5,35 metros e DAP de $11,4 \mathrm{~cm}$.

A espécie A. cearensis é definida como clímax (SIQUEIRA FILHO et al., 2009), por isso apresenta características de crescimento lento. Assim, devido ao ritmo muito lento de crescimento de espécies clímax, mesmo havendo condições ambientais favoráveis, de acordo com Souza et al. (1993), a duração do crescimento de espécies clímax pode chegar a vários séculos. Com isso, se faz necessário o conhecimento dos processos de dinâmica de crescimento para se tenha um correto manejo das florestas nativas.

Dessa forma, a necessidade de conservação da espécie A. cearensis tem se tornando urgente, no qual medidas conservacionistas precisam ser adotadas devido ao seu risco de extinção, e a falta de conhecimento sobre o seu crescimento lento, associado ao extrativismo predatório, facilita esse processo de supressão da espécie. Portanto, essas informações das características de crescimento de Amburana cearensis auxilia na escolha de técnicas adequadas para manejo da espécie para fins de conservação.

Carvalho (2003) cita a importância da conservação da Amburana cearensis ex situ, e que a mesma está sendo conservada pela Embrapa. Segundo Brasil (2008), é uma das espécies prioritárias para conservação de germoplasma, devido a mesma está ameaçada de extinção.

Durante o período de um ano, foi observada a mortalidade de 43 mudas (33\%). Isso se deve provavelmente a baixa frequência de irrigação, realizada apenas uma vez ao mês, o que pode ter ocasionado a morte de alguns acessos. Além disso, devido as mudas apresentarem um crescimento lento, característica típica de espécies clímax, ou seja, espécies de final de sucessão ecológica, as condições ambientais locais (escassez de chuvas, altas temperaturas e área de implantação com ausência de vegetação pioneira), pode ter comprometido a sobrevivência dessas mudas.

\section{CONCLUSÃO}

As sementes da espécie Amburana cearensis apresentam um alto índice de germinação sem necessidade de tratamentos físico-químicos, confirmando que não há dormência na espécie em estudo, portanto, não apresentando dificuldades para a formação de mudas, o que facilita o plantio em larga escala para conservação da espécie. No entanto, a espécie apresenta crescimento lento, o que a caracteriza como uma espécie clímax.

Considerando que a espécie $A$. cearensis apresenta múltiplos usos, é de grande importância a conservação deste recurso genético, pois pelo fato dessa espécie apresentar crescimento muito lento, a exploração insustentável desse recurso vegetal facilitaria sua extinção. A implantação de bancos 
ativos de germoplasma, torna-se ambientalmente necessário e socialmente justificável, como também o desenvolvimento de outras atividades florestais para o manejo sustentável e programas para conservação da espécie. Além disso, esse estudo também busca sensibilizar a sociedade sobre a preservação da espécie.

\section{REFERÊNCIAS}

ANGELIM, A.E.S. et al. Germinação e aspectos morfológicos de plantas de umburana de cheiro (Amburana cearensis) encontradas na região do Vale do São Francisco. Revista Brasileira de Biociências, v. 5, p. 1062- 1064, 2007.

BRASIL. Ministério do Meio Ambiente. Instrução Normativa nº. 06, de 23 de setembro de 2008. Lista Oficial da flora brasileira ameaçada de extinção. Diário Oficial [da] República Federativa do Brasil, Brasília, DF, n. 185, p. 75-83, 24 set. 2008.

CANUTO, K.M. et al. Phytochemistry, pharmacology and agronomy of medicinal plants: Amburana cearensis, an interdisciplinary study. In: RAO, V. (Ed.). Phytochemicals: A global perspective of their role in nutrition and health. Rijeka: InTech, 2012. p. 353-374.

CARVAlHO, P.E.R. Cumaru. Brasília, DF: Embrapa Informação Tecnológica; Colombo, PR: Embrapa Florestas, 2003. 11 p. (Circular Técnica, 76).

DANTAS, B.F. et al. Produção de mudas de catingueira-verdadeira (Caesalpinia pyramidalis Tul.) em função de substratos e luminosidades. Científica, v. 39, n. 1, p. 34-43, 2011.

FIGUEREDO, F.G. et al. Modulation of the antibiotic activity by extracts from Amburana cearensis A. C. Smith and Anadenanthera macrocarpa (Benth.) Brenan. BioMed Research International, v. 2013, p. 1-5.

GUEDES, R.S. et al. Emergência e vigor de plântulas de Amburana cearensis (Allemão) A.C. Smith em função da posição e da profundidade de semeadura. Semina: Ciências Agrárias, v. 31, n. 4, p. 843-850, 2010a.

GUEDES, R.S. et al. Qualidade fisiológica de sementes armazenadas de Amburana cearensis (Allemão) A.C. Smith. Semina: Ciências Agrárias, v. 31, n. 2, p. 331-342, 2010b.

GUEDES, R.S. et al. Avaliação do potencial fisiológico de sementes de Amburana cearensis (Allemão) A.C. Smith. Bioscience Journal, v. 29, n. 4, p. 859-866, 2013.

GUEDES, R.S. et al. Teste de comprimento de plântula na avaliação da qualidade fisiológica de sementes de Amburana cearensis (Allemão) A.C. Smith. Semina: Ciências Agrárias, v. 36, n. 4, p. 2373-2381, 2015.

LEAL, L.K.A.M. et al. A comparative chemical and pharmacological study of standardized extracts and vanillic acid from wild and cultivated Amburana cearensis A.C. Smith. Phytomedicine, v. 18, p. 230-233, 2011. 
LIMA, L.R. et al. Avaliação da atividade antiedematogênica, antimicrobiana e mutagênica das sementes de Amburana cearensis (A. C. Smith) (Imburana-de-cheiro). Revista Brasileira de Plantas Medicinais, v. 15, n. 3, p. 415-422, 2013.

LOPES, I.S. et al. Maturação e colheita da semente de Amburana cearensis (Allem.) A.C. Smith. Ciência Florestal, v. 24, n. 3, p. 565-572, 2014.

LORENZI, H. Árvores brasileiras: manual de identificação e cultivo de plantas arbóreas nativas do Brasil. 5. ed. Nova Odessa, SP: Editora Plantarum, 2008. 384 p.

LORENZI, H.; MATOS, F.J.A. Plantas medicinais no Brasil: nativas e exóticas. 2. ed. Nova Odessa, SP: Instituto Plantarum, 2008. 544 p.

MAIA, G.N. Caatinga: árvores e arbustos e suas utilidades. 1. ed. São Paulo: D \& Z Editora, 2004. $413 \mathrm{p}$.

MARTINS, C.C. et al. Efeito da posição da semente no substrato e no crescimento inicial das plântulas de palmito-vermelho (Euterpe espiritusantensis Fernandes Palmae). Revista Brasileira de Sementes, v. 21, n. 1, p. 164-173, 1999.

OLIVEIRA, G.M. et al. Germinação de sementes de espécies arbóreas nativas da Caatinga em diferentes temperaturas. Scientia Plena, v. 10, n. 4, p. 1-6, 2014.

OLIVEIRA, R.R.B. et al. Antinociceptive effect of the ethanolic extract of Amburana cearensis (Allemão) A.C. Sm., Fabaceae, in rodents. Revista Brasileira de Farmacognosia, v. 19, n. 3, p. 672-676, 2009.

RAMOS, K.M.O. et al. Desenvolvimento inicial e repartição de biomassa de Amburana cearensis (Allemao) A.C. Smith, em diferentes condições de sombreamento. Acta botanica Brasilica, v. 18, $\mathrm{n}$. 2, p. 351-358, 2004.

SIQUEIRA FILHO, J.A. et al. Guia de Campo de Árvores da Caatinga. Petrolina: Editora e Gráfica Franciscana, 2009. 64 p.

SOUZA, A.L. et al. Dinâmica de crescimento em diâmetro de uma floresta primária sem interferência: uma análise pelo tempo de passagem entre classes diamétricas. Revista Árvore, v. 17, n. 2, p. 129-145, 1993. 